\title{
On the Convergence of a Finite Element Method for a Nonlinear Hyperbolic Conservation Law
}

\author{
By Claes Johnson and Anders Szepessy
}

\begin{abstract}
We consider a space-time finite element discretization of a time-dependent nonlinear hyperbolic conservation law in one space dimension (Burgers' equation). The finite element method is higher-order accurate and is a Petrov-Galerkin method based on the so-called streamline diffusion modification of the test functions giving added stability. We first prove that if a sequence of finite element solutions converges boundedly almost everywhere (as the mesh size tends to zero) to a function $u$, then $u$ is an entropy solution of the conservation law. This result may be extended to systems of conservation laws with convex entropy in several dimensions. We then prove, using a compensated compactness result of Murat-Tartar, that if the finite element solutions are uniformly bounded then a subsequence will converge to an entropy solution of Burgers' equation. We also consider a further modification of the test functions giving a method with improved shock capturing. Finally, we present the results of some numerical experiments.
\end{abstract}

0. Introduction. In this note we prove some results concerning the convergence of a higher-order accurate finite element method for a nonlinear hyperbolic conservation law. As far as we know, no earlier theoretical results for such methods are available. We shall consider Burgers' equation in one space dimension, i.e., the problem of finding a scalar function $u(x, t)$ such that

$$
\begin{gathered}
u_{t}+u u_{x}=0, \quad x \in R=(-\infty, \infty), t>0, \\
u(0, x)=u_{0}(x), \quad x \in R,
\end{gathered}
$$

where $u_{0}$ is a given function with, say, compact support. As is well known, solutions of (0.1) may become nonsmooth and develop shocks after finite time, even for smooth initial data $u_{0}$. Further, weak solutions of $(0.1)$ are not necessarily unique, and to guarantee uniqueness, one has to require a certain entropy condition to be satisfied. A weak solution satisfying the entropy condition is the (unique) so-called entropy solution of (0.1).

We shall consider a special finite element method for $(0.1)$, namely the streamline diffusion method based on a space-time finite element discretization with piecewise polynomials of degree $k>0$, together with a certain modification of the test functions giving added stability. We shall first prove that if a sequence of finite element solutions converges (as the mesh size $h$ tends to zero) boundedly almost everywhere to a function $u$, then $u$ is an entropy solution of $(0.1)$. Thus, streamline diffusion finite element solutions cannot converge to a weak solution not satisfying

Received March 6, 1986; revised September 15, 1986.

1980 Mathematics Subject Classification (1985 Revision). Primary 65M15.

Key words and phrases. Finite element method, convergence, nonlinear hyperbolic conservation law. 
the entropy condition, i.e., to a nonphysical solution. The method may directly be formulated for systems of conservation laws in several dimensions and the convergence result just stated extends to this situation if so-called entropy variables are used (see [12]).

We shall further prove that if the streamline diffusion finite element solutions are uniformly bounded, then a subsequence will converge almost everywhere to a function $u$. We thus conclude that if the finite element solutions are uniformly bounded, then a subsequence will converge to an entropy solution of (0.1). This result is obtained by an application of the theory of compensated compactness, introduced by Murat and Tartar (see [15]) and exploited by DiPerna [1]. We emphasize that our finite element method is higher-order accurate (for smooth solutions the error is $O\left(h^{k+1 / 2}\right)$ ) and is not based on adding heavy artificial viscosity, limiting the accuracy to at best $O(h)$. The problem of proving uniform boundedness for the finite element solutions is left open (no maximum principle seems to be available). However, it may in fact be possible to prove convergence without the uniform boundedness assumption ( $\mathrm{R}$. DiPerna, personal communication; cf. also Remark 4.4 below).

Streamline diffusion type finite element methods (under the name SUPG, Streamline Upwind Petrow Galerkin) were first applied to nonlinear (stationary) hyperbolic conservation laws in Hughes and Tezduyar [3] with further development in Hughes et al. [4]-[8]. The advantage of using entropy variables in finite element formulations was pointed out in [4], [5], with inspiration from, e.g., Harten [2] and Tadmor [14]. In the cited work by Hughes et al. several very good numerical results for streamline methods for the stationary compressible Euler equations in two dimensions are reported.

Note that we have earlier ([9], [10], [11]) demonstrated the satisfactory properties, including good stability and high accuracy of streamline diffusion finite element methods, for linear advection problems, in particular for nonsmooth exact solutions, and for the incompressible Euler and Navier-Stokes equations. Conventional finite element methods lack in either stability (as standard Galerkin methods) or accuracy (as classical artificial viscositý methods). In particular, with a standard Galerkin method for (0.1) there is a possibility of convergence to a nonphysical solution not satisfying the entropy condition.

Although the streamline diffusion method in its basic form discussed so far gives a dramatic improvement as compared to the standard Galerkin method, still some overshoots persist at discontinuities of the exact solution. Recently, in the case of stationary problems, Hughes et al. [6], [8] introduced a variant of the streamline method, a shock-capturing streamline method, based on a certain ingeniously chosen additional modification of the test functions and demonstrated in numerical experiments the improved shock resolution of the modified method.

In this note we also consider a shock-capturing modification of the basic streamline diffusion method for Burgers' equation obtained by applying the idea of [6] to the present time-dependent problem. The resulting scheme indeed shows considerably improved shock resolution in numerical tests. The convergence results for the basic streamline method stated above carry over also to the shock-capturing modification, but the problem of mathematically analyzing the improved shock resolution of the modified scheme is open, even for linear problems. 
We finally present the results of some numerical experiments for Burgers' equation without and with shock-capturing, demonstrating a very satisfactory performance in the latter case.

Let us recall that the basic convergence results for finite difference methods for scalar conservation laws are based on a priori estimates for first derivatives in $L_{1}$-norms (BV-estimates). Such estimates seem to be very difficult to obtain for finite element methods on general meshes, and thus the basic theory for finite difference methods for scalar conservation laws can most likely not be extended to finite element methods. However, for systems of conservation laws one can probably not expect to achieve $L_{1}$-control of first derivatives (cf. [13]), and thus a new approach to the convergence problem may have to be made anyway in this case. Here, the theory of compensated compactness may open possibilities of proving convergence without derivative estimates, cf. [1], [15].

To sum up, the theoretical and computational results for the streamline method obtained so far indicate that efficient finite element methods may be designed for hyperbolic conservation laws (in particular, if coupled with adaptively constructed meshes). Thus, in compressible flow there seems to exist a finite element alternative to the up till now dominating $L_{1}$ or BV based finite difference methodology.

1. The Streamline Diffusion Method. Let us now define the basic streamline diffusion method for (0.1). Let $0=t_{0}<t_{1}<t_{2} \cdots$ be a sequence of time levels, set $I_{n}=\left(t_{n}, t_{n+1}\right)$ and introduce the 'slabs' $S_{n}=R \times I_{n}$. For $h>0$ and $n=0,1,2, \ldots$ let $T_{h}^{n}$ be a quasi-uniform triangulation of $S_{n}$ into triangles $K$ of diameter $h_{K} \leqslant h$ with smallest angle uniformly bounded away from zero, and define for a given $k \geqslant 1$,

$$
V_{h}^{n}=\left\{v \in H^{1}\left(S_{n}\right):\left.v\right|_{K} \in P_{k}(K), K \in T_{h}^{n}\right\},
$$

where $P_{k}(K)$ denotes the set of polynomials on $K$ of degree at most $k$. Typically, $t_{n+1}-t_{n} \sim h$ and thus $S_{n}$ will normally be one element wide, cf. Section 6 . Note that since $u_{0}$ has compact support, it follows that also the solution $u$ has compact support in $R \times[0, t]$ for any $t$. This means that we may restrict the functions in $V_{h}^{n}$ to be zero for $|x|$ large.

We shall seek an approximate solution $U \equiv U_{h}$ in the space $V_{h}=\prod_{n \geqslant 0} V_{h}^{n}$, i.e., for $n=0,1,2, \ldots$, we will have

$$
\left.U\right|_{S_{n}} \in V_{h}^{n}
$$

Note that the functions in $V_{h}$ are continuous in $x$ and possibly discontinuous in $t$ at the discrete time levels $t_{n}$. The streamline diffusion method for $(0.1)$ can now be formulated as follows: Find $U \in V_{h}$ such that for $n=0,1,2 \ldots$,

$$
\int_{S_{n}}\left(U_{t}+U U_{x}\right)\left(v+h\left(v_{t}+U v_{x}\right)\right) d x d t+\int_{R}\left(U_{+}^{n}-U_{-}^{n}\right) v_{+}^{n} d x=0
$$

$\forall v \in V_{h}^{n}$,

where $\delta=h$ (cf. Remark 4.5) and

$$
v_{ \pm}^{n}=\lim _{s \rightarrow 0^{ \pm}} v\left(t_{n}+s\right), \quad U_{-}^{0}=u_{0} .
$$


As in [10] we see that (1.1) admits at least one solution. Note that (1.1) corresponds to an implicit time stepping scheme; see Section 6 below.

Our main concern is now the problem of convergence of the method (1.1), i.e., whether $U_{h}$ converges in a suitable sense to an entropy solution $u$ of $(0.1)$ as $h$ tends to zero. To make this precise, let us recall that a function $u \in L_{\infty}(\Omega), \Omega=R \times$ $(0, \infty)$, is a weak solution of $(0.1)$ if for all $\varphi \in C_{0}^{\infty}(\bar{\Omega}), \bar{\Omega}=R \times[0, \infty)$, we have

$$
\int_{\Omega}\left(u \varphi_{t}+\frac{u^{2}}{2} \varphi_{x}\right) d x d t+\int_{R} u_{0} \varphi(\cdot, 0) d x=0 .
$$

Further, we recall ([1], [15]) that a weak solution $u$ of $(0.1)$ is an entropy solution if the following entropy inequality is satisfied: For all $\varphi \in C_{0}^{\infty}(\Omega)$ with $\varphi \geqslant 0$,

$$
\int_{\Omega}\left[\frac{u^{2}}{2} \varphi_{t}+\frac{u^{3}}{3} \varphi_{x}\right] d x d t \geqslant 0
$$

Thus, our problem is to prove that $U_{h}$ converges to a function $u$ satisfying (1.2) and (1.3).

Remark. An entropy solution of Burgers' equation according to the above definition is most likely unique. However, a proof of uniqueness seems to be available in the literature only under the additional assumption that $u$ has bounded variation, see [1].

We will use the following notation: For $\omega$ a domain in $R^{d}, d=1,2$, and $m$ a nonnegative integer, let $H^{m}(\omega)$ denote the usual Sobolev space of functions with derivatives up to order $m$ square integrable over $\omega$, with norm $\|\cdot\|_{m, \omega}$ and corresponding seminorm $|\cdot|_{m, \omega}$ including only highest-order derivatives. Further, if $m=0$, this index is omitted, so that $\|\cdot\|_{\omega}=\|\cdot\|_{L_{2}(\omega)}$. Also, we write $\|\cdot\|_{\infty, \omega}=$ $\|\cdot\|_{L_{x}(\omega)}$. We also use the convention that for $N \geqslant 1$

$$
\left\|U_{t}+U U_{x}\right\|_{\Omega_{N}}^{2}=\sum_{n=0}^{N-1}\left\|U_{t}+U U_{x}\right\|_{S_{n}}^{2}
$$

where $\Omega_{N}=R \times\left(0, t_{N}\right)$. By $C$ we will denote a positive constant independent of $u_{0}$ and $h$, not necessarily the same at each occurrence.

2. Preliminaries. A Basic Estimate. The basic stability estimate for (1.1) is obtained by taking $v=U$, which gives by integration by parts and summation over $n$ : For $N=1,2, \ldots$, we have

$$
h\left\|U_{t}+U U_{x}\right\|_{\Omega_{N}}^{2}+\frac{1}{2} \sum_{n=0}^{N-1}\left\|U_{+}^{n}-U_{-}^{n}\right\|_{R}^{2}+\frac{1}{2}\left\|U_{-}^{N}\right\|_{R}^{2}=\frac{1}{2}\left\|u_{0}\right\|_{R}^{2} .
$$

We also note that from (2.1) we may obtain an estimate for $\|U(t)\|_{R}$ for all $t>0$ as follows: For $t_{n}<t<t_{n+1}$ we have

$$
\begin{aligned}
\|U(t)\|_{R}^{2} & =\left\|U_{-}^{n+1}\right\|_{R}^{2}-\int_{t}^{t_{n+1}} \frac{d}{d s}\|U(s)\|_{R}^{2} d s \\
& =\left\|U_{-}^{n+1}\right\|_{R}^{2}-2 \int_{t}^{t_{n+1}} \int_{R}\left(U_{t}+U U_{x}\right) U d x d s \\
& \leqslant\left\|U_{-}^{n+1}\right\|_{R}^{2}+h\left\|U_{t}+U U_{x}\right\|_{S_{n}}^{2}+\frac{1}{h} \int_{t}^{t_{n+1}}\|U(s)\|_{R}^{2} d s
\end{aligned}
$$


so that by Gronwall's inequality for $t_{n}<t<t_{n+1}$,

$$
\|U(t)\|^{2} \leqslant C\left[h\left\|U_{t}+U U_{x}\right\|_{S_{n}}^{2}+\left\|U_{-}^{n+1}\right\|_{R}^{2}\right] .
$$

Together with (2.1) this proves that

$$
\|U(t)\|_{R} \leqslant C\left\|u_{0}\right\|_{R}, \quad t>0 .
$$

Further, with $\pi_{h} v \in V_{h}$ a standard interpolate of $v \in H^{1}(\Omega)$, we have for $s=0,1$ the interpolation error estimates

$$
\begin{gathered}
\left\|v-\pi_{h} v\right\|_{s, \Omega} \leqslant C h^{m-s}|v|_{m, \Omega}, \quad 1 \leqslant m \leqslant k+1, \\
\left\|v_{+}^{n}-\left(\pi_{h} v\right)_{+}^{n}\right\|_{R} \leqslant C \sqrt{h}|v|_{1, S_{n} .}
\end{gathered}
$$

We also have the following "super-approximation" result:

Lemma 2.1. There is a constant $C$ such that for $\varphi \in H^{1}(\Omega), v \in V_{h}, n=0,1,2, \ldots$,

$$
\begin{gathered}
\left\|v \varphi-\pi_{h}(v \varphi)\right\|_{s, \Omega} \leqslant C h^{1-s}\|v\|_{L^{\infty}(\Omega)}\|\varphi\|_{1, \Omega}, \quad s=0,1, \\
\left\|(v \varphi)_{+}^{n}-\left(\pi_{h}(v \varphi)\right)_{+}^{n}\right\|_{R} \leqslant C \sqrt{h}\|v\|_{L^{\infty}(\Omega)}\|\varphi\|_{1, S_{n}} .
\end{gathered}
$$

Proof. To prove (2.4a), it is sufficient to prove that for each triangle $K \in T_{h}^{n}$,

$$
\left\|v \varphi-\pi_{h}(v \varphi)\right\|_{s, K} \leqslant C h^{1-s}\|v\|_{\infty, K}\|\varphi\|_{1, K}, \quad s=0,1 .
$$

To this end, let $\varphi_{h} \in P_{0}(K)$ be defined by

$$
\int_{K} \varphi_{h} w d x=\int_{K} \varphi w d x \quad \forall w \in P_{0}(K)
$$

i.e., $\varphi_{h}$ is the $L_{2}(K)$-projection of $\varphi$ onto the set $P_{0}(K)$ of constants on $K$. By well-known estimates we have

$$
\left\|\varphi-\varphi_{h}\right\|_{K} \leqslant C h\|\varphi\|_{1, K}
$$

Further,

$$
\begin{aligned}
\left\|v \varphi-\pi_{h}(v \varphi)\right\|_{K} & \leqslant\left\|v \varphi_{h}-\pi_{h}\left(v \varphi_{h}\right)\right\|_{K}+\left\|v \varphi-v \varphi_{h}\right\|_{K}+\left\|\pi_{h}(v \varphi)-\pi_{h}\left(v \varphi_{h}\right)\right\|_{K} \\
& =T_{1}+T_{2}+T_{3}
\end{aligned}
$$

with obvious notation. Now, since $v \in V_{h}$ and $\varphi_{h}$ is constant on $K, \pi_{h}\left(v \varphi_{h}\right)=v \varphi_{h}$ on $K$ and thus $T_{1}=0$. Further, using (2.5), we have

$$
T_{2}=\left\|v \varphi-v \varphi_{h}\right\|_{K} \leqslant C h\|v\|_{\infty, K}\|\varphi\|_{1, K},
$$

and

$$
\begin{aligned}
T_{3} & \leqslant\left\|\pi_{h}\left(v\left(\varphi-\varphi_{h}\right)\right)\right\|_{K} \\
& \leqslant\left\|v\left(\varphi-\varphi_{h}\right)\right\|_{K}+\left\|v\left(\varphi-\varphi_{h}\right)-\pi_{h}\left(v\left(\varphi-\varphi_{h}\right)\right)\right\|_{K} \\
& \leqslant C h\|v\|_{\infty, K}\|\varphi\|_{1, K}+C h\left|v\left(\varphi-\varphi_{h}\right)\right|_{1, K} \leqslant C h\|v\|_{\infty, K}\|\varphi\|_{1, K},
\end{aligned}
$$

where in the last step we also used the inverse estimate

$$
|v|_{W_{\infty}^{1}(K)} \leqslant C h^{-1}|v|_{L_{\infty}(K)}, \quad v \in P_{k}(K) .
$$

Recalling (2.6) and (2.7), we thus obtain (2.4a) with $s=0$. In a similar way we obtain (2.4a) with $s=1$ and (2.4b), and the lemma follows. 
3. Convergence Towards Entropy Solutions. We start our discussion with the following result, which proves that if the solutions $U_{h}$ of (1.1) converge boundedly almost everywhere to a function $u$, then $u$ satisfies (1.2) and (1.3), i.e., $u$ is an entropy solution of (0.1). As noted, this proves that the method (1.1) cannot produce discrete solutions converging to a nonphysical solution of $(0.1)$, or in other words, that (1.1) in some sense has a correct dissipation built in. Again we note that this dissipative effect is not obtained by adding a strong dissipative term of, e.g., the form $-h u_{x x}$ to $(0.1 \mathrm{a})$, as is typically done in the case of monotone difference schemes (e.g., the Lax-Friedrichs' scheme). Such an added dissipative term limits the order of accuracy to at most first order, while the scheme (1.1) is of order at least $k+1 / 2$ for smooth solutions.

THEOREM 3.1. Suppose the solutions $U_{h}$ of (1.1) converge boundedly a.e. in $\Omega$ to a function $u$ as $h$ tends to zero, so that in particular for $h>0$,

$$
\left\|U_{h}\right\|_{\infty, \Omega} \leqslant C \text {. }
$$

Then $u$ satisfies (1.2) and (1.3), and thus $u$ is an entropy solution of (0.1).

Proof. To prove that $u$ satisfies (1.2), we choose $v=\pi_{h} \varphi$ with $\varphi \in C_{0}^{\infty}(\bar{\Omega})$ in (1.1) to get

$$
\begin{aligned}
\int_{S_{n}}\left(U_{t}+\right. & \left.U U_{x}\right) \varphi d x d t+\int_{R}\left(U_{+}^{n}-U_{-}^{n}\right) \varphi_{+}^{n} d x \\
= & \int_{S_{n}}\left(U_{t}+U U_{x}\right)\left(\varphi-\pi_{h} \varphi\right) d x d t+\int_{R}\left(U_{+}^{n}-U_{-}^{n}\right)\left(\varphi_{+}^{n}-\left(\pi_{h} \varphi\right)_{+}^{n}\right) d x \\
& -h \int_{S_{n}}\left(U_{t}+U U_{x}\right)\left(\left(\pi_{h} \varphi\right)_{t}+U\left(\pi_{h} \varphi\right)_{x}\right) d x d t \\
= & E_{n}^{1}+E_{n}^{2}+E_{n}^{3},
\end{aligned}
$$

with the obvious notation. Integrating here by parts on the left-hand side and summing over $n$, we get

$$
\begin{aligned}
-\int_{\Omega}\left(U \varphi_{t}\right. & \left.+\frac{U^{2}}{2} \varphi_{x}\right) d x d t-\int_{R} u_{0} \varphi(\cdot, 0) d x \\
= & \sum_{n \geqslant 0} E_{n}^{1}+\sum_{n \geqslant 0} E_{n}^{2}+\sum_{n \geqslant 0} E_{n}^{3} \equiv R_{h}^{1}+R_{h}^{2}+R_{h}^{3} .
\end{aligned}
$$

Now, by (2.3), (2.1) and (3.1) we have

$$
\begin{aligned}
& \left|R_{h}^{i}\right| \leqslant C h\left\|U_{t}+U U_{x}\right\|_{\Omega}\|\varphi\|_{1, \Omega} \leqslant C \sqrt{h}\|\varphi\|_{1, \Omega}, \quad i=1,3, \\
& \left|R_{h}^{2}\right| \leqslant C \sqrt{h}\left(\sum_{n \geqslant 0}\left\|U_{+}^{n}-U_{-}^{n}\right\|_{R}^{2}\right)^{1 / 2}\|\varphi\|_{1, \Omega} \leqslant C \sqrt{h}\|\varphi\|_{1, \Omega} .
\end{aligned}
$$

Passing to the limit in (3.2), we now see that $u$ satisfies (1.2). 
Finally, to prove (1.3), we choose $v=\pi_{h}(U \varphi)$ with $\varphi \geqslant 0, \varphi \in C_{0}^{\infty}(\Omega)$ to get

$$
\begin{aligned}
\int_{S_{n}}( & \left.U_{t}+U U_{x}\right) U \varphi d x d t+\int_{R}\left(U_{+}^{n}-U_{-}^{n}\right) U_{+}^{n} \varphi_{+}^{n} d x+h \int_{S_{n}}\left(U_{t}+U U_{x}\right)^{2} \varphi d x d t \\
= & \int_{S_{n}}\left(U_{t}+U U_{x}\right)\left(U \varphi-\pi_{h}(U \varphi)\right) d x d t \\
& +\int_{R}\left(U_{+}^{n}-U_{-}^{n}\right)\left((U \varphi)_{+}^{n}-\left(\pi_{h}(U \varphi)\right)_{+}^{n}\right) d x \\
& +h \int_{S_{n}}\left(U_{t}+U U_{x}\right)\left((U \varphi)_{t}-\left(\pi_{h}(U \varphi)\right)_{t}+U\left((U \varphi)_{x}-\left(\pi_{h}(U \varphi)\right)_{x}\right)\right) d x d t \\
& -h \int_{S_{n}}\left(U_{t}+U U_{x}\right)\left(U \varphi_{t}+U^{2} \varphi_{x}\right) d x d t \equiv F_{n}^{1}+F_{n}^{2}+F_{n}^{3}+F_{n}^{4},
\end{aligned}
$$

again with the obvious notation. Integrating by parts as above and summing over $n$, we get

$$
\begin{aligned}
-\int_{\Omega}\left(\frac{U^{2}}{2} \varphi_{t}+\frac{U^{3}}{3} \varphi_{x}\right) d x & d t+\frac{1}{2} \sum_{n \geqslant 1} \int_{R}\left(U_{+}^{n}-U_{-}^{n}\right)^{2} \varphi_{+}^{n} d x \\
& +h \int_{\Omega}\left(U_{t}+U U_{x}\right)^{2} \varphi d x d t=\sum_{j=1}^{4} \sum_{n \geqslant 0} F_{n}^{j} \equiv \sum_{j=1}^{4} S_{h}^{j} .
\end{aligned}
$$

Using now (2.1), (2.4) and (3.1), we conclude that

$$
\begin{gathered}
\left|S_{h}^{j}\right| \leqslant C h\left\|U_{t}+U U_{x}\right\|_{\Omega}\|\varphi\|_{1, \Omega} \leqslant C \sqrt{h}\|\varphi\|_{1, \Omega}, \quad j=1,3,4, \\
\left|S_{h}^{2}\right| \leqslant C \sqrt{h}\left(\sum_{n \geqslant 0}\left\|U_{+}^{n}-U_{-}^{n}\right\|_{R}\right)^{1 / 2}\|\varphi\|_{1, \Omega} \leqslant C \sqrt{h}\|\varphi\|_{1, \Omega} .
\end{gathered}
$$

Passing then to the limit in (3.3), we find that $u$ satisfies (1.3), and the proof is complete.

Remark 3.1. Theorem 3.1 may be extended to systems of conservation laws in several dimensions using entropy variables; see [12].

4. Convergence a.e. by Compensated Compactness. We now relax the conditions of Theorem 3.1 and only assume that the maximum norm bound (3.1) is satisfied. Under this assumption we shall prove, using a compensated compactness result by Murat and Tartar (see [15]), that a subsequence $U_{h}$ converges a.e. to a function $u$. By Theorem 3.1 it then follows that $u$ is an entropy solution of $(0.1)$. We thus conclude that a subsequence $U_{h}$ converges a.e. towards an entropy solution of (0.1) if assumption (3.1) is satisfied.

THEOREM 4.1. Suppose the solutions $U_{h}$ of (1.1) satisfy (3.1). Then a subsequence $U_{h}$ converges a.e. to an entropy solution $u$ of (0.1).

Proof. According to [5] it is sufficient to prove that

$$
\frac{\partial}{\partial t} \eta(U)+\frac{\partial}{\partial x} q(U) \in A+B
$$

where $U=U_{h}$ and

$$
\begin{aligned}
& A=\text { compact set of } H^{-1}(\Omega), \\
& B=\text { bounded set of } M(\Omega),
\end{aligned}
$$


with the following two choices of the functions $\eta$ and $q$ :

$$
\begin{aligned}
\eta(v) & =v, & q(v) & =\frac{v^{2}}{2}, \\
\eta(v) & =\frac{v^{2}}{2}, & q(v) & =\frac{v^{3}}{3} .
\end{aligned}
$$

Here, $M(\Omega)$ denotes the set of bounded measures on $\Omega$.

To prove (4.1) in the case (4.2), let us choose $v=\pi_{h} \varphi, \varphi \in C_{0}^{\infty}(\Omega)$ in (1.1) to give as above

$$
\begin{aligned}
-\int_{\Omega}( & \left.U \varphi_{t}+\frac{U^{2}}{2} \varphi_{x}\right) d x d t \\
= & \int_{\Omega}\left(U_{t}+U U_{x}\right)\left(\varphi-\pi_{h} \varphi\right) d x d t+\sum_{n \geqslant 1} \int_{R}\left(U_{+}^{n}-U_{-}^{n}\right)\left(\varphi_{+}^{n}-\left(\pi_{h} \varphi\right)_{+}^{n}\right) d x \\
& -h \int_{\Omega}\left(U_{t}+U U_{x}\right)\left(\left(\pi_{h} \varphi\right)_{t}+U\left(\pi_{h} \varphi\right)_{x}\right) d x d t \\
\leqslant & C h\left\|U_{t}+U U_{x}\right\|_{\Omega}\|\varphi\|_{1, \Omega}+C \sqrt{h}\left(\sum_{n \geqslant 0}\left\|U_{+}^{n}-U_{-}^{n}\right\|_{R}^{2}\right)^{1 / 2}\|\varphi\|_{1, \Omega},
\end{aligned}
$$

so that by $(2.1)$

$$
\left|\int_{\Omega}\left(U \varphi_{t}+\frac{U^{2}}{2} \varphi_{x}\right) d x d t\right| \leqslant C \sqrt{h}\|\varphi\|_{1, \Omega}
$$

This proves that

$$
\frac{\partial}{\partial t} \eta(U)+\frac{\partial}{\partial x} q(U) \in A
$$

and (4.1) follows in the case (4.2).

Finally, in the case (4.3) we choose in (1.1) $v=\pi_{h}(U \varphi), \varphi \in C_{0}^{\infty}(\Omega)$, to get as above

$$
\begin{aligned}
-\int_{\Omega}\left(\frac{U^{2}}{2} \varphi_{t}\right. & \left.+\frac{U^{3}}{3} \varphi_{x}\right) d x d t \\
& =-h \int_{\Omega}\left(U_{t}+U U_{x}\right)^{2} \varphi d x d t-\frac{1}{2} \sum_{n \geqslant 0} \int_{R}\left(U_{+}^{n}-U_{-}^{n}\right)^{2} \varphi_{+}^{n} d x+\sum_{j=1}^{4} S_{h}^{j},
\end{aligned}
$$

so that, recalling (2.1) and (3.4),

$$
\left|\int_{\Omega}\left(\frac{U^{2}}{2} \varphi_{t}+\frac{U^{3}}{3} \varphi_{x}\right) d x d t\right| \leqslant C\|\varphi\|_{L^{\infty}(\Omega)}+C \sqrt{h}\|\varphi\|_{1, \Omega} .
$$

This proves (4.1) in the case (4.3), and the proof is complete.

Remark 4.1. Notice that since the flux function $f(u)=u^{2} / 2$ in Burgers' equation is strictly convex, it is sufficient to establish (4.1) for one strictly convex entropy $\eta$ and corresponding entropy flux $q$, e.g., with $\eta$ and $q$ given by (4.3) (cf. [15]). For a general nonconvex $f$ we would have to prove (4.1) for all convex entropies $\eta$ and corresponding fluxes $q$, a considerably more difficult task. 
Remark 4.2. In the compensated compactness argument that we have used, the assumption of one space dimension seems essential. Thus, extension of Theorem 4.1 to the case of several space dimensions is not direct.

Remark 4.3. We notice that the entropy condition (1.3) for Burgers' equation used in this note corresponds to the entropy condition for the compressible Euler equations expressed in entropy variables used in [4], [5]. In both cases the entropy condition is basically obtained by multiplying the conservation law itself by the quantity $u \varphi$, where $u$ is the unknown and $\varphi$ a nonnegative test function, and then integrating. From the finite element point of view, this is a favorable situation, since the discrete counterpart of the entropy inequality can then be obtained by taking $v=\pi_{h}(U \varphi)$ in the finite element equation; cf. [12].

Remark 4.4. The assumption (3.1) is used at two crucial steps above, namely in (4.4)-(4.5) and in the Murat-Tartar result used in the proof of Theorem 4.1. In both cases the assumption (3.1) may be replaced by a somewhat weaker condition; in (4.5) we only need that $\sqrt{h}\left\|U_{h}\right\|_{\infty, \Omega}^{2}$ tends to zero as $h$ tends to zero, and to apply the Murat-Tartar result, it seems to be possible to replace (3.1) by the assumption $\left\|U_{h}\right\|_{L_{p}(\Omega)} \leqslant C$ for some $p>6$.

Remark 4.5. To obtain the theoretical results of this paper, it is not criticial to use precisely the test functions $\left(v+h\left(v_{t}+U v_{x}\right)\right)$ in (1.1). In fact, it is sufficient to choose $\delta=\delta(h)$ such that $\delta \rightarrow 0$ and $h / \sqrt{\delta} \rightarrow 0$ as $h \rightarrow 0$. The choice $\delta=h$ (or more precisely $\delta \sim\left(1+U^{2}\right)^{-1 / 2} h$, cf. [4], [12]), however, seems most natural and is also the choice that gives $O\left(h^{k+1 / 2}\right)$ convergence for smooth solutions; cf. Section 6 below.

5. A Shock-Capturing Streamline Diffusion Method. We now consider a variant of the streamline diffusion method (1.1) with improved shock-capturing (cf. [6], [8]). Using the notation

$$
\beta=\beta(U)=(1, U), \quad \nabla v=\left(v_{t}, v_{x}\right),
$$

the modified test function in (1.1) can be written

$$
v+\delta\left(v_{t}+U v_{x}\right)=v+\delta \beta \cdot \nabla v
$$

where $\beta \cdot \nabla v=v_{t}+U v_{x}$.

The further modified test functions in the shock-capturing streamline diffusion method are given by

$$
v+\delta \beta \cdot \nabla v+\bar{\delta} \bar{\beta} \cdot \nabla v
$$

where $\bar{\beta}=\bar{\beta}(U)$ is the projection of $\beta(U)$ onto $\nabla U$ (locally for each $(x, t)$ ), that is,

$$
\bar{\beta}=\frac{\beta \cdot \nabla U}{|\nabla U|^{2}} \nabla U,
$$

and $\bar{\delta}$ is a parameter again with $\bar{\delta} \sim h$. Note that by the definition of $\bar{\beta}$, we have

$$
\bar{\beta} \cdot \nabla U=\beta \cdot \nabla U .
$$


The shock-capturing streamline diffusion method can now be formulated as follows: Find $U \in V_{h}$ such that for $n=0,1,2, \ldots$,

$$
\begin{array}{r}
\int_{S_{n}}\left(U_{t}+U U_{x}\right)(v+\delta \beta(U) \cdot \nabla v+\bar{\delta} \bar{\beta}(U) \cdot \nabla v) d x d t \\
+\int_{R}\left(U_{+}^{n}-U_{-}^{n}\right) v_{+}^{n} d x=0, \quad \forall v \in V_{h}^{n} .
\end{array}
$$

Theorems 3.1 and 4.1 directly extend to the shock-capturing method (5.2). Note that the basic stability estimate for (5.2), in view of (5.1), is the same as (2.1) (with $h$ replaced by $(\delta+\bar{\delta})$ ). As already indicated, the improved shock-capturing of (5.2) still awaits analysis.

6. Numerical Results. We now give some numerical results obtained using the obvious version of (1.1) and (5.2) with piecewise bilinear finite elements on a uniform mesh of the form

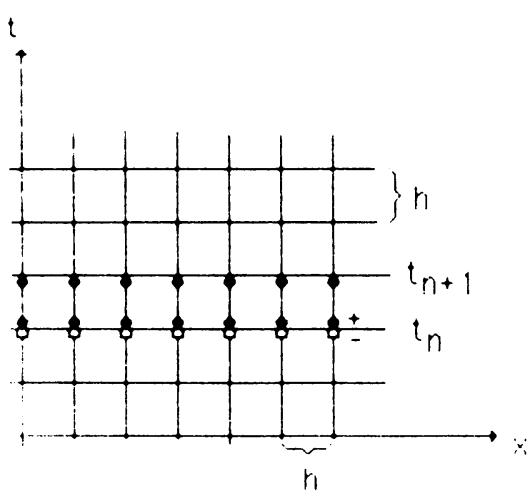

For each $n$ we have in this case that (1.1) and (5.2) is equivalent to a nonlinear system of equations with unknowns representing the nodal values of $U_{+}^{n}$ and $U_{-}^{n+1}$ at the points marked by solid circles in the above figure. Here, also the known nodal values of $U_{-}^{n}$ entering (1.1) as data are indicated by squares. The computations were made with $h=0.1$ and with the following initial data:

$$
u_{0}(x)= \begin{cases}0 & \text { for } x<2.05 \\ 1 & \text { for } 2.05<x \leqslant 5 \\ (7.9-x) / 2.9 & \text { for } 5 \leqslant x \leqslant 7.9 \\ 0 & \text { for } x \geqslant 7.9\end{cases}
$$

The corresponding exact solution has a rarefaction wave centered at $x=2.05$ and develops a shock at $x=7.9$ at time $t=2.9$.

Below we give the results after 1, 21 and 51 time steps obtained using (1.1) with $\delta=h$, and (5.2) with $\delta=h$ and $\bar{\delta}=h, 2.5 h$ and $5 h$. The exact solution is represented by the dashed line. We note the improved shock-resolution of (5.2) with proper choice of $\bar{\delta}$. For comparison we also give the results at the corresponding time levels obtained by the Lax-Friedrichs' finite difference method and Godunov's method with $\Delta t=\Delta x=0.1$ and initial data $=\frac{1}{2} u_{0}$. 


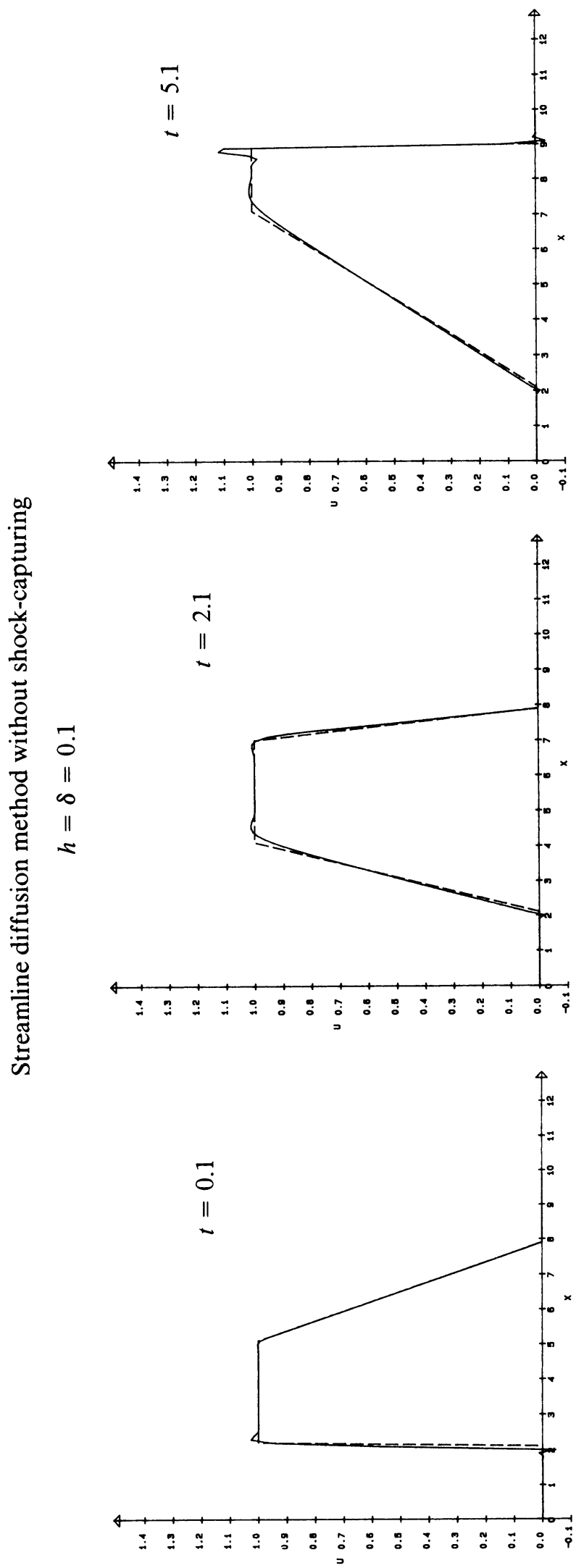



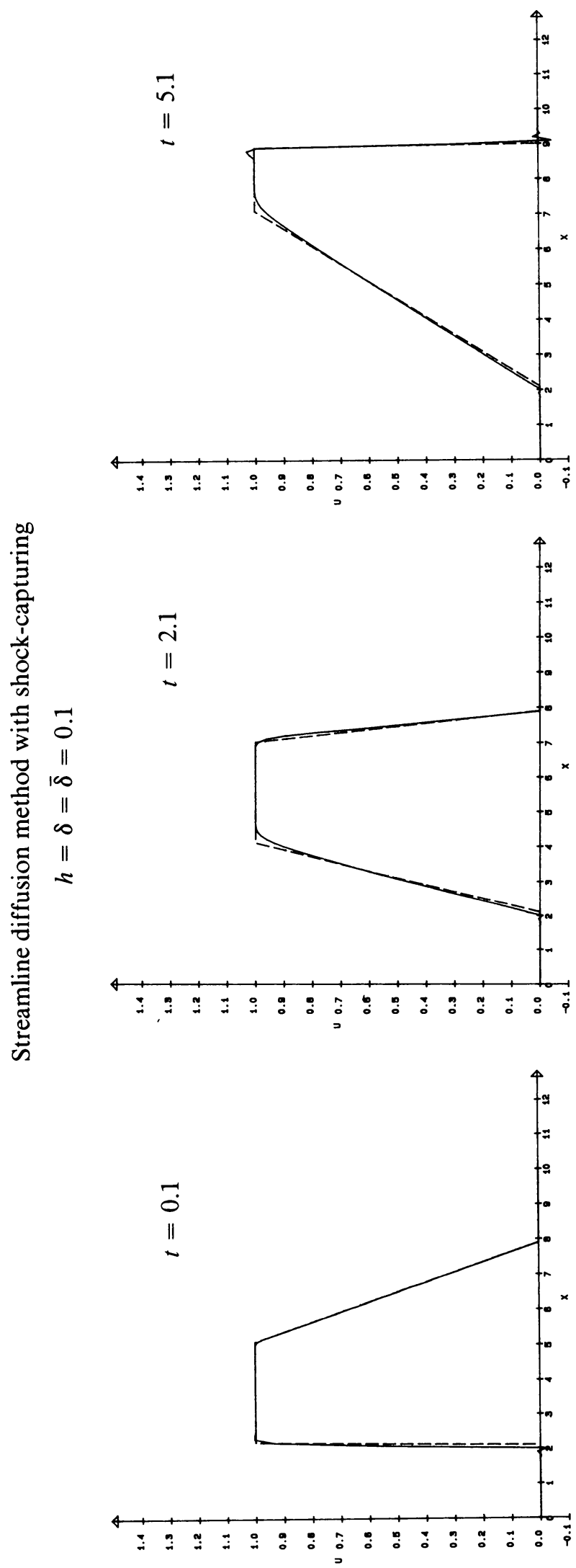

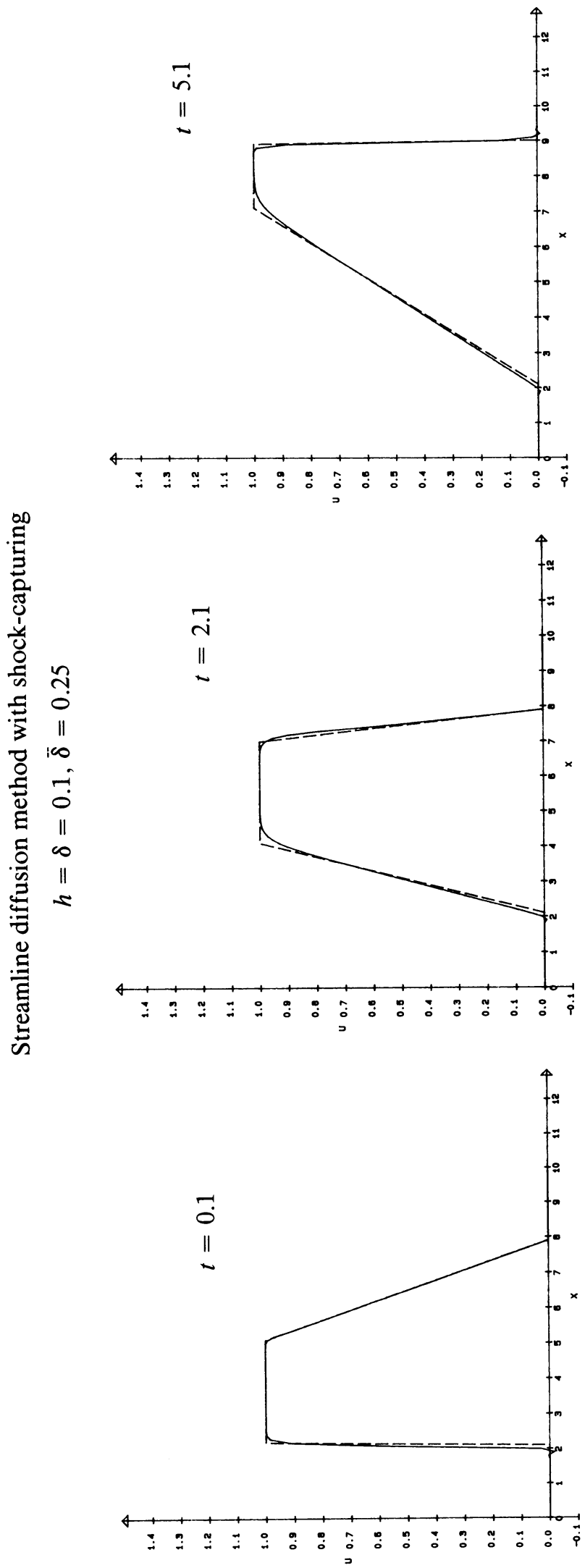

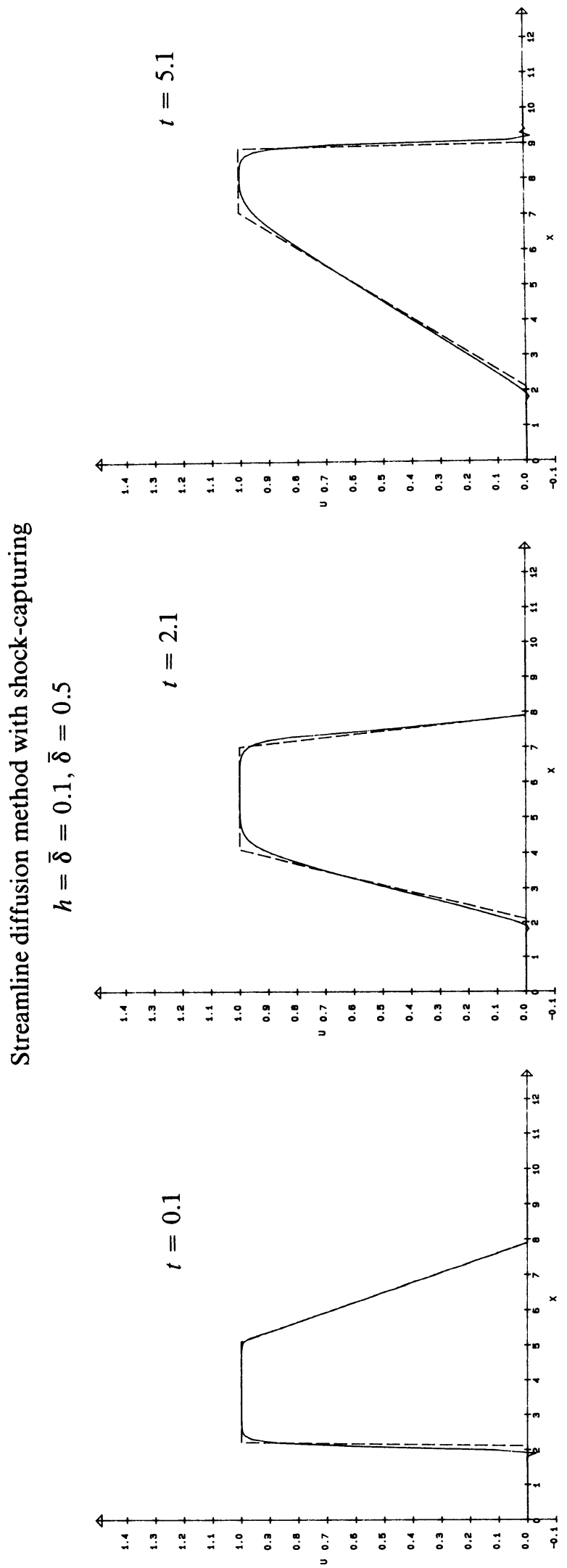

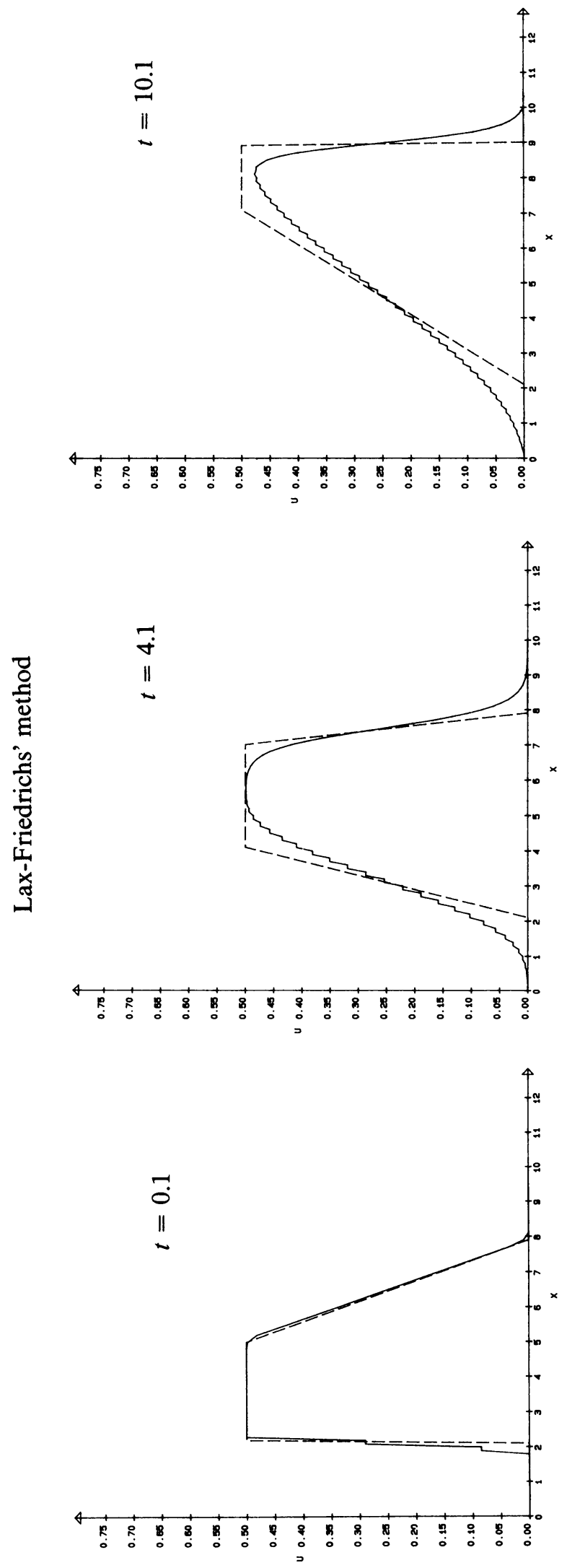

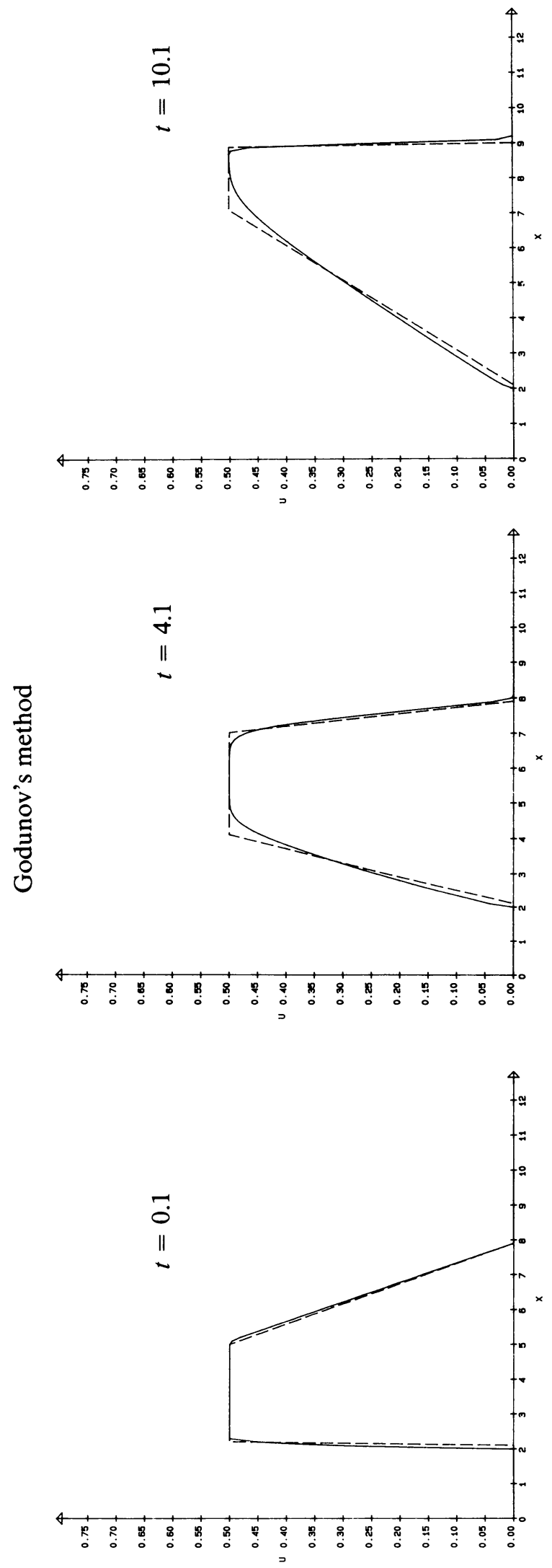


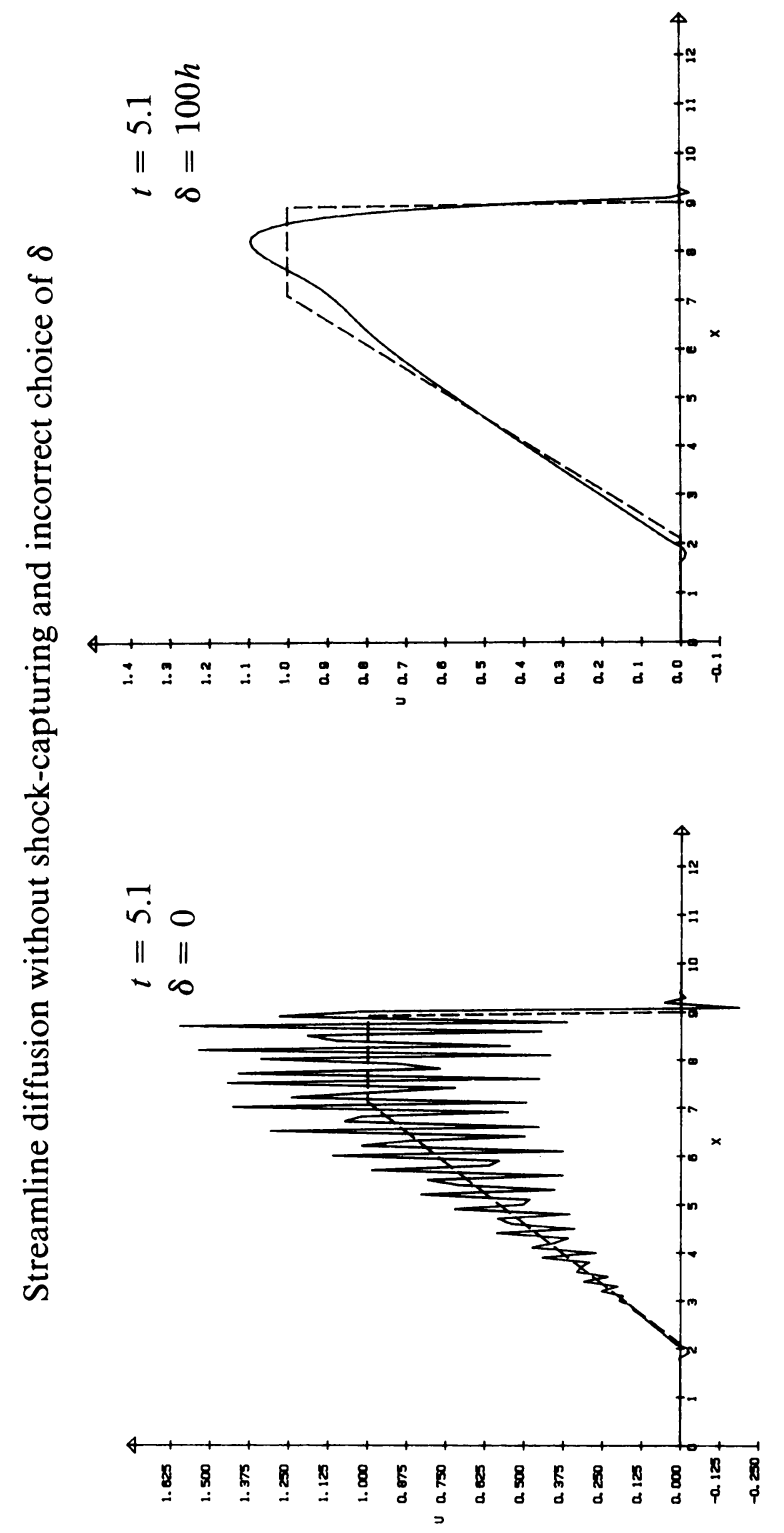


Department of Mathematics

Chalmers University of Technology

and The University of Göteborg

41296 Göteborg, Sweden

1. R. J. DiPerna, "Convergence of approximate solutions to conservation laws," Arch. Rational Mech. Anal., v. 82, 1983, pp. 27-70.

2. A. HaRten, "On the symmetric form of systems of conservation laws with entropy," J. Comput. Phys., v. 49, 1983, pp. 151-164.

3. T. J. R. Hughes \& T. E. Tezduyar, "Finite element methods for first-order hyperbolic systems with particular emphasis on the compressible Euler equations," Comput. Methods Appl. Mech. Engrg., v. 45, 1984, pp. 217-284.

4. T. J. Hughes, M. Mallet \& L. P. Franca, "Entropy stable finite element methods for compressible fluids: Application to high Mach number flows with shocks," in Finite Elements for Nonlinear Problems (P. Bergen, K. J. Bathe and W. Wunderlich, eds.), Springer, Berlin, 1986, pp. $761-773$.

5. T. J. R. Hughes, L. P. Franca \& M. Mallet, "A new finite element formulation for computational fluid dynamics: I. Symmetric forms of the compressible Euler and Navier-Stokes equations and the second law of thermodynamics," Comput. Methods Appl. Mech. Engrg., v. 54, 1986, pp. 223-234.

6. T. J. R. Hughes, M. Mallet \& A. Mizukami, "A new finite element method for computational fluid dynamics: II. Beyond SUPG,” Comput. Methods Appl. Mech. Engrg., v. 54, 1986, pp. 341-355.

7. T. J. Hughes \& M. Mallet, "A new finite element formulation for computational fluid dynamics: III. The generalized streamline operator for multi-dimensional advective-diffusive systems," Comput. Methods Appl. Mech. Engrg., v. 58, 1986, pp. 305-328.

8 T. J. Hughes \& M. MAllet, "A new finite element formulation for computational fluid dynamics: IV. A discontinuity capturing operator for multidimensional advective-diffusive systems," Comput. Methods Appl. Mech. Engrg., v. 58, 1986, pp. 329-336.

9. C. Johnson, U. Nävert \& J. Pitkäranta, "Finite element methods for linear hyperbolic problems," Comput. Methods Appl. Mech. Engrg., v. 45, 1985, pp. 285-312.

10. C. Johnson \& J. SARANEN, "Streamline diffusion methods for the incompressible Euler and Navier-Stokes equations," Math. Comp., v. 47, 1986, pp. 1-18.

11. C. JoHnson, "Streamline diffusion methods for problems in fluid mechanics," in Finite Elements in Fluids, vol. 6 (Gallagher et al., eds.), Wiley, New York, 1985.

12. C. Johnson \& A. SZEPESSY, On the Convergence of Streamline Diffusion Finite Element Methods for Hyperbolic Conservation Laws, Numerical Methods for Compressible Flows-Finite Difference, Element and Volume Techniques-AMD vol. 78 (T. E. Tezduyar and T. J. R. Hughes, eds.).

13. J. RaUCH, BV Estimates Fail for Most Quasilinear Hyperbolic Systems in Dimensions Greater Than One, Technical report, École Polytechnique, Palaiseau, 1986.

14. E. TADMOR, "Skew-selfadjoint forms for systems of conservation laws," J. Math. Anal. Appl., v. 103,1984 , pp. $428-442$.

15. L. TARTAR, "Compensated compactness and applications to partial differential equations," in Research Notes in Mathematics, Nonlinear Analysis and Mechanics: Heriot-Watt Symposium, Vol. 4 (R. J. Knops, ed.), Pitman Press, London, 1979. 